\title{
Scanning Probe Microscopic Studies of the Oriented Attachment and Membrane Reconstitution of Cytochrome $c$ Oxidase to a Gold Electrode
}

\author{
Dirk Mayer $,{ }^{*}, \dagger$ Kenichi Ataka,,+ Joachim Heberle,$\stackrel{\ddagger}{\ddagger}$ and Andreas Offenhäusser ${ }^{\dagger}$ \\ Research Center Juelich, ISG-2: Institute for Bio and Chemosensors, \\ IBI-2: Structural Biology, D-52425 Juelich, Germany \\ Received May 4, 2005. In Final Form: July 17, 2005
}

\begin{abstract}
Scanning probe microscopy was used to monitor the resulting surface of the oriented incorporation of cytochrome $c$ oxidase into electrode supported lipid bilayer at four crucial stages with molecular resolution. We were able to reveal the formation of a densely packed monolayer of the active ester dithio(succiniimidylepropionate) (DTSP) and the covalent linkage of the nitrilotriacetic acid (NTA) to the thiol anchored DTSP by scanning tunneling microscopy. Atomic force microscopy investigations showed that the detergent solubilized oxidase is immobilized as monomers and small aggregates via histidine residues. Finally, the reconstitution of the proteins within the supported membrane was verified. The amount of oxidase immobilized within the solid supported membrane was estimated.
\end{abstract}

\section{Introduction}

Many of the vital functions of cells are maintained by membrane proteins, which for instance selectively control the transfer of ions, functional agents, and energy between the periplasm and cytoplasm of a cell. Often, a certain function is accomplished by the complex interplay of a number of different proteins supported by a defined environment. The high complexity of biological transmembrane machineries with respect to their structure, the reduced periodicity and the multistep reaction sequences makes the assignment of structure and function a challenging task. The reconstitution of membrane proteins on functional solid surfaces such as electrodes is required in order to develop novel analytical in vivo strategies to further enlighten the factors that control the protein-based machineries. The oriented reconstitution of proteins without denaturation is a prerequisite for deriving reliable information and still represents a major experimental challenge.

Recently, some of us (Ataka and Heberle) succeeded in tethering the membrane protein cytochrome $c$ oxidase $(\mathrm{CcO})$ to the surface of a gold electrode and in reconstituting the protein into a lipid layer. ${ }^{1}$ In the cell, this protein is embedded in the inner mitochondrial membrane. $\mathrm{CcO}$ is the terminal enzyme of the respiratory chain that catalyzes the reduction of a molecule of dioxygen to two molecules of water, consuming four protons and four electrons provided by reduced cytochrome $c$ (cyt-c) molecules. The free energy made available by this reaction is coupled to the translocation of four additional protons. ${ }^{2}$ The net movement of charges contributes to the electrochemical gradient that is used to support the adenosine triphosphate production performed by the $\mathrm{H}^{+}$-ATP-synthase.

We developed a novel approach to tether membrane proteins to the solid surface which combines two common

* To whom correspondence should be addressed. Phone: +49 246161 4023. Fax: +49 246161 8733. E-mail: dirk.mayer@ fz-juelich.de.

Research Center Juelich ISG-2: Institute for Bio and Chemosensors.

$\doteqdot$ Research Center Juelich IBI-2: Structural Biology.

(1) Ataka, K.; Giess, F.; Knoll, W.; Naumann, R.; Haber-Pohlmeier, S.; Richter, B.; Heberle, J. J Am Chem Soc. 2004, 126, 16199-16206.

(2) Wikström, M. Biochim. Biophys. Acta 2004, 1655, 241-247. experimental concepts. ${ }^{1}$ At first, the metal surface is modified by attaching molecules with $\mathrm{Ni}^{2+}$ ion chelated nitrilotriacetic acid (Ni-NTA) headgroup via a sulfhydryl group at the other end. Recombinant membrane protein engineered to bear a stretch of six consecutive histidine residues (His-tag) at the $\mathrm{C}$ - or the $\mathrm{N}$-terminus, which was purified and solubilized in detergent, is attached by the affinity to the Ni- NTA moiety. ${ }^{3-5}$ Second, the oriented surface attached protein is reconstituted into a lipid environment by in situ dialysis. ${ }^{6}$ In our previous work, ${ }^{1}$ we have employed surface-enhanced infrared absorption spectroscopy (SEIRAS) to investigate each modification step during the immobilization of $\mathrm{CcO}$ along the solid surface of gold. In this work, we applied scanning probe microscopy in order to derive the 2-dimensional surface structure at each step of the reconstitution of cytochrome $c$ oxidase at the electrode. These images provide direct evidence for the microscopic architecture of the surfacetethered protein. In particular, the AFM/STM experiments reveal (1) the formation of a densely packed monolayer of the active ester, dithio(succiniimidylepropionate) (DTSP), which is a template layer for following cross-linking reaction, (2) the covalent linkage of the NTA to the thiol anchored DTSP with almost $100 \%$ yield, (3) the detergent solubilized oxidase is immobilized in mono- and oligomeric form, and (4) the reconstitution of the proteins into the lipid layer of di-myristoyl-phosphatidyl-choline (DMPC).

\section{Materials and Methods}

For all preparations, $\mathrm{Au}(111)-(1 \times 1)$ was used as the substrate surface. The gold single crystal was annealed in a $\mathrm{H}_{2-}$ flame and cooled to room temperature (within $\sim 5 \mathrm{~min}$ ) in an argon atmosphere. ${ }^{7}$ Details of the surface modification steps have been described elsewhere. ${ }^{1}$ Briefly, the bare gold surface was immersed in a solution of $1 \mathrm{mg} / \mathrm{mL}$ dithiobis(succiniimidylpropionate) (DTSP, Fluka) in dimethyl sulfoxide (DMSO, Fluka) for $15 \mathrm{~min}$. The disulfide bridge of DTSP is cleaved by contact with

(3) Hochuli, E.; Bannwarth, W.; Dobeli, H.; Gentz, R.; Stuber, D. Biotechnology 1988, 6, 1321-1325.

(4) Kröger, D.; Liley, M.; Schiweck, W.; Skerra, A.; Vogel, H. Biosens. Bioelectron. 1999, 14, 155-161.

(5) Winzerling, J. J.; Berna, P.; Porath, J. Methods: Companion Methods Enzymol. 1992, 4, 4-13.

(6) Singh, S.; Keller, D. J. Biophys J. 1991, 60, 1401-1410.

(7) Hölzle, M.H.; Wandlowski, Th.; Kolb, D. M. J. Electroanal. Chem. 1995, 394, 271. 


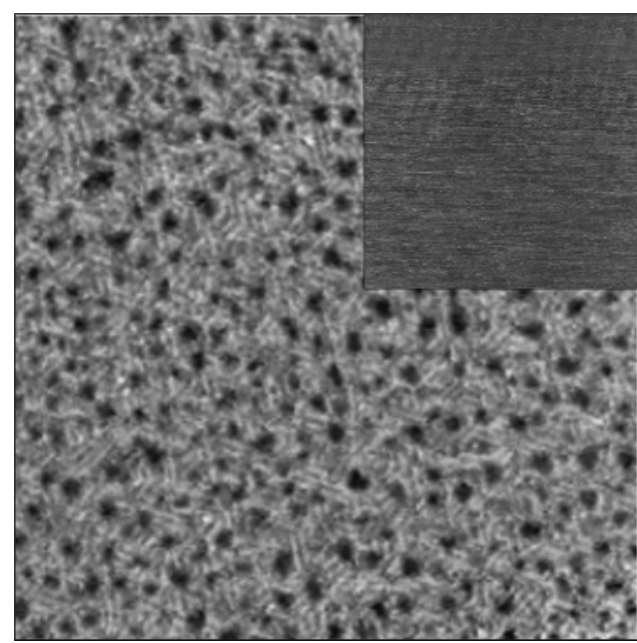

Figure 1. Ex situ STM image of $A u(111)-(1 \times 1)$ surface modified by a self-assembled monolayer of the heterobifunctional cross-linker thiosuccinimidyl- propionate (TSP), $E_{\text {bias }}=$ $80 \mathrm{mV}, I_{\mathrm{T}}=30 \mathrm{pA}$, scan size $90 \mathrm{~nm}$. The insert shows the bare $\mathrm{Au}(111)-(1 \times 1)$ surface before TSP adsorption, $E_{\text {bias }}=25 \mathrm{mV}$, $I_{\mathrm{T}}=840 \mathrm{pA}$, scan size $110 \mathrm{~nm}$.

the gold surface. The resulting thiosuccinimidyl-propionate (TSP) forms a monolayer spontaneously with the metal surface through covalent linkage to the sulfur group. In the second reaction step, the freshly prepared self-assembled TSP monolayer is immersed for $1 \mathrm{~h}$ in an aqueous solution of $150 \mathrm{mM} N_{\alpha}{ }^{\prime}, N_{\alpha}{ }^{\prime \prime}$-bis(carboxymethyl)-L-lysine or aminonitrilotriacetic acid (ANTA, Fluka) and $0.5 \mathrm{M} \mathrm{K}_{2} \mathrm{CO}_{3}$ ( $\mathrm{pH}$ 9.8). The amino-group of ANTA reacts with the thiosuccinimidyl-propionate to form a carboxamide linkage. ${ }^{8}$ Successively, the NTA terminated surface was incubated for 15 min with $50 \mathrm{mM} \mathrm{NiSO}_{4}$ (Sigma-Aldrich) solution to ligate the $\mathrm{Ni}^{2+}$ ion via the three carboxylates and the tertiary amine of NTA.

After complexation, $2 \mu \mathrm{M} \mathrm{CcO}$ dissolved in $0.1 \%$ dodecylmaltoside (DM, Anatrace, solgrade 3105) and $50 \mathrm{mM}$ phosphate buffer $(\mathrm{pH}=8.0)$ was added to the Ni-NTA coated Au surface for $1 \mathrm{~h}$. Homologous expression of $\mathrm{CcO}$ in Rhodobacter sphaeroides and purification has been described elsewhere. ${ }^{9,10}$ The reconstitution of the enzyme into a lipid layer was carried out by overnight incubation of surface-tethered $\mathrm{CcO}$ in a solution containing detergent-destabilized lipid vesicles (liposomes) of di-myristoylphosphatidyl-choline (DMPC from Sigma) and Bio-Beads for detergent removal. ${ }^{6}$

All images were acquired at room temperature with a MultiMode AFM/STM (Veeco/Digital Instruments, Santa Barbara, CA) equipped with a Nanoscope IV controller and a $15 \mu \mathrm{m}$ scanner. The STM tips were electrochemically etched from a $0.25 \mathrm{~mm} \mathrm{Pt}(70) / \mathrm{Ir}(30)$ (imaging at ambient conditions) or tungsten wires. The latter were coated with polyethylene for measurements in solution. AFM measurements were performed in tapping mode. Commercially available silicon cantilevers have been employed with resonance frequencies between 260 and $300 \mathrm{kHz}$.

\section{Results and Discussion}

The atomically flat $\mathrm{Au}(111)-(1 \times 1)$ surface (insert Figure 1) of a flame annealed gold single crystal has been incubated with DTSP to form a self-assempled monolayer (SAM) of covalently bound TSP. The surface morphology of the TSP layer was characterized by STM experiments under ambient conditions (ex situ). Figure 1 presents a closely packed monolayer of the heterobifunctional crosslinker TSP. Each bright spot is representative for a specifically arranged assembly of a few adsorbed TSP

(8) Hermanson, G. T. Bioconjugate techniques; Academic Press: San Diego, CA, 1996.

(9) Mitchell, D. M.; Gennis, R. B. FEBS Lett. 1995, 368, 148-150.

(10) Nyquist R. M.; Heitbrink D.; Bolwien C.; Wells T. A.; Gennis R.

B.; Heberle J. FEBS Lett. 2001, 505, 63-67. molecules. Only short range order could be observed with an average domain size of $5 \times 5 \mathrm{~nm}$. The domains comprise about 3-5 molecular rows. Three rotational domains are present in the STM images, rotated by angles of $60^{\circ}$ and $120^{\circ}$, reflecting the symmetry of the underlying $\mathrm{Au}(111)-$ $(1 \times 1)$ surface. Several pit-like defects are observed that are surrounded by these ordered molecular layers. The depth of the pit-like defects is $2.5 \AA$, which is commonly found for thiol based SAMs and assigned to 2D vacancy islands in the topmost gold layer. ${ }^{11-13}$ The molecules are assembled to rowlike structures with a mean length and width of $5 \mathrm{~nm}( \pm 2 \mathrm{~nm})$ and $1.4 \mathrm{~nm}( \pm 0.4 \mathrm{~nm})$, respectively. Besides these molecular layers, one can observe several domains where the molecular order is distorted. The dimensions deviate from the mean value by several $\AA$ at the vicinity of defects and distorted areas.

The self-assembled and densely packed TSP monolayer provides the succinimidyl functionality for the subsequent surface cross-linking. The structure after the second reaction step, where ANTA molecules are coupled to the TSP monolayer, was investigated by STM in electrolyte solution under potential control (in situ). The potential of the sample and of the imaging tungsten tip was controlled by a bipotentiostat. All potentials are quoted with respect to the saturated calomel electrode (SCE). Formation of carboxamide (-CONH-) bonds due to the cross-linking between ANTA and TSP layer were confirmed by IR spectroscopy in our previous work. ${ }^{1}$ The in situ STM experiments of the NTA-functionalized surface reveal a densely packed monolayer as shown in Figure 2a. Again, the surface shows the typical vacancy islands of thiolbased SAM. Furthermore, we observed rowlike molecular structures as in the upper case of the TSP-modified surface. The mean length of the stripes is $10 \mathrm{~nm}( \pm 1 \mathrm{~nm})$, but rows with a length of up to $20 \mathrm{~nm}$ can also be found. The mean width is determined to $1.6 \mathrm{~nm}( \pm 0.2 \mathrm{~nm})$. The experiments reveal an increased long range order and smaller deviations in the dimensions, as compared to the TSP-modified surfaces. The size of the molecular domains is limited by the appearance of vacancy islands. The mean domain size was determined to $10 \mathrm{~nm} \times 10 \mathrm{~nm}$. Three different domains are discernible in the STM images, which are $120^{\circ}$ rotated with respect to each other, induced by the underlying gold (111) surface. The resolution of the in situ STM image is sufficient to observe individual molecules (see bright spots in Figure 2b) with a mean distance between the molecules of $5.3 \AA( \pm 0.4 \AA)$. The detailed structural model of the arrangement of the NTA-monolayer will be proposed in a forthcoming publication. ${ }^{14}$ However, it is reasonable to conclude that the dimensions of the molecular structures of the TSP and the NTA-modified surface are quite similar since the succinimidyl-ester moiety provides the anchor for the linkage of the ANTA. The NTA-moiety matches the structural template of the rigid TSP layer due to the strong $\mathrm{Au}-\mathrm{S}$ bonds and the highly organized and associated molecular assembly. The slightly increased width of the molecular rows can be explained by the higher sterical expansion of the ANTA molecule as compared to the succinimidyl moiety.

The Ni-NTA modified surface is ready to bind recombinant cytochrome $c$ oxidase $(\mathrm{CcO})$ via the coordination of the nitrogens of two of the imidazole side chains of the $6 \mathrm{x}$ His-tag. $\mathrm{CcO}$ adsorbs to the Ni-NTA-cross-linker in an

(11) Petri, M.; Kolb, D. M.; Memmert U.; Meyer H. Electrochim. Acta 2003, 49, 175-182.

(12) Poirier, G. E. Chem. Rev. 1997, 97, 1117-1127.

(13) Poirier, G. E. Langmuir 1997, 13, 2019-2026.

(14) Mayer, D.; Ataka, K.; Heberle, J.; Offenhäusser, A. Unpublished work, 2005 . 
a

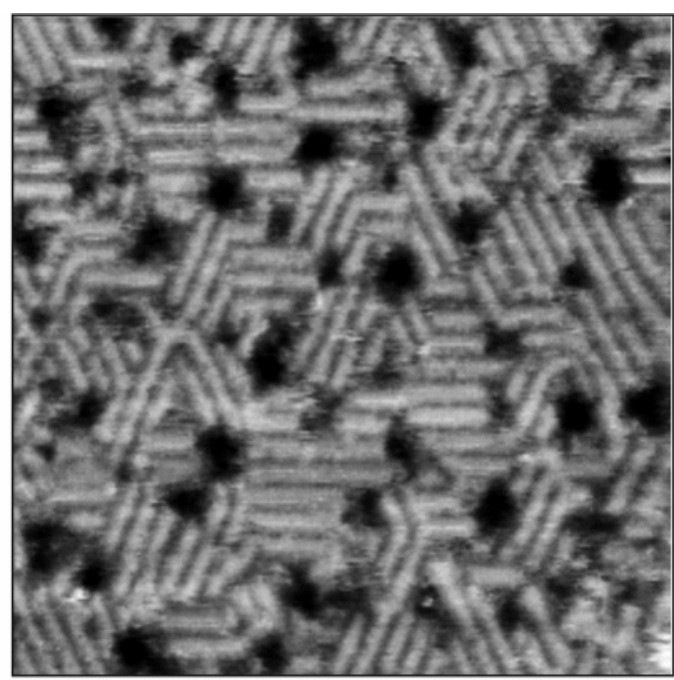

b

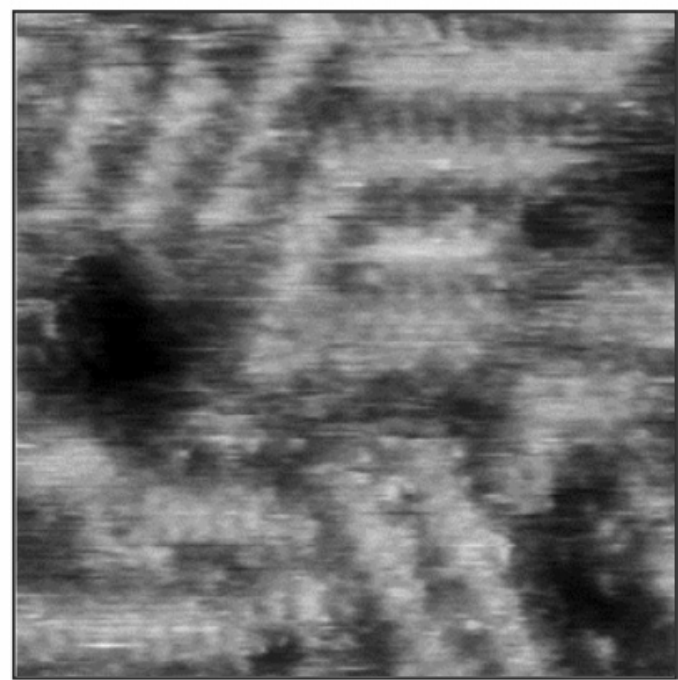

Figure 2. In situ STM images of the aminonitrilotriacetic acid (NTA) self-assembled monolayer linked to the thiol-anchored $\mathrm{TSP}, E_{\text {bias }}=40 \mathrm{mV}, E_{\text {sample }}=-30 \mathrm{mV}, I_{\mathrm{T}}=70 \mathrm{pA}$, (a) scan size $50 \mathrm{~nm}$ and (b) scan size $13 \mathrm{~nm}$.

oriented fashion such that the cytochrome $c$ binding site is freely accessible to the solution, since the His-tag is engineered into the C-terminus of subunit I. The attachment of the enzymes was previously demonstrated by the appearance of amide I and the amide II modes of the amino acid backbone vibrations in FTIR spectra. ${ }^{1}$

The investigation of the morphology of the surface immobilized $\mathrm{CcO}$ was performed at ambient conditions. The topographical image (Figure $3 \mathrm{a}$ ) reveals closely packed and laterally agglomerated features with height variations in the range of $4-10 \mathrm{~nm}$ and a diameter that ranges from 15 to $30 \mathrm{~nm}$. The adsorbed $\mathrm{CcO}$ covers the entire surface as a monolayer. There was no indication for the formation of multilayers. Hemispherical features with a mean width of $7 \mathrm{~nm}( \pm 2 \mathrm{~nm})$ are protruded by $1-2 \mathrm{~nm}$ at some of the aggregates (Figure 3b). Similar protruded objects are described already as cone-shaped features by Burgess and co-workers for bovine $\mathrm{CcO}$ incorporated into an alkanethiol supported lipid bilayer. ${ }^{15}$ The diameter of the $1-2 \mathrm{~nm}$ high protuberances and the height of the agglomerates actually correspond to the diameter of the $\mathrm{CcO}$. We conclude from both the shape and the determined sizes of the surface features in relation to the findings of the

(15) Burgess, J. D.; Jones, V. W.; Porter, M. D.; Rhoten, M. C.; Hawkridge, F. M. Langmuir 1998, 14, 6628-6631.
SEIRAS experiments that the TM-AFM images represent monolayers of solubilized $\mathrm{CcO}$. The monolayer is composed of individual proteins as well as proteins that are integrated into larger (lateral) aggregates. The enzyme is wrapped by a detergent shell which presumably fuses for aggregated $\mathrm{CcO}$ molecules and covers them entirely. Individual dimers and higher aggregated proteins were reported also for platinum/carbon replicas of freezefractured vesicles investigated by electron microscopy. ${ }^{16}$ The high specificity of CcO binding to the Ni-NTA layer through the His-tag was verified by the detachment of $\mathrm{CcO}$ from $\mathrm{Ni}-\mathrm{NTA}$ by adding imidazole. ${ }^{1}$

Finally, the oriented, surface attached $\mathrm{CcO}$ is reconstituted in a lipid environment by in situ dialysis. ${ }^{6}$ It is expected that the restoration into a physiologically natural matrix enhances the stability of the membrane protein and preserves its functional integrity. The presence of Bio-Beads induces the slow retreat of detergent surrounding of the enzyme. The lipid vesicles spread over the surface and spontaneously form a bilayer that embed the surface tethered membrane protein. Previous IR experiments revealed that there was no indication of any change in the adsorbed protein layer during the dialysis process. ${ }^{1}$

The TM-AFM investigations of the lipid modified surface reveal formation of the bilayer, whereas some parts are covered with a multilayer structure (Figure 4c). The thickness of each layer was determined by measuring both the height of the second bilayer and depth of a broader pinhole to $5.2 \mathrm{~nm}( \pm 0.5 \mathrm{~nm})$, which is a commonly found value for DMPC lipid bilayers. ${ }^{1,17}$ About $40 \%$ of the overall surface is covered by just a single bilayer membrane. Separated proteins can be observed as well as individual proteins that stick together (Figure 4a). Consequently, the findings for the reconstituted $\mathrm{CcO}$ confirm the derived model of individual and agglomerated detergent solubilized enzymes of the previous section. The large aggregates (Figure 3a) disappear during the reconstitution process. The mean diameter of the individual proteins is determined to $7.2 \mathrm{~nm}( \pm 1.5 \mathrm{~nm})$, which is in accordance to the diameter of a single cytochrome $c$ oxidase. The protein coverage in the first bilayer was estimated to $33 \%( \pm 4 \%)$ with respect to the gold surface area. This corresponds to $1.3 \mathrm{pmol} / \mathrm{cm}^{2}$ of $\mathrm{CcO}$ assuming a protein size of $8 \mathrm{~nm} \times$ $6 \mathrm{~nm}$. The enzyme is $1 \mathrm{~nm}( \pm 0.2 \mathrm{~nm})$ protruded from the lipid membrane beside minor height fluctuations between the $\mathrm{CcO}$. The same kind of minor corrugations (smaller than $0.5 \mathrm{~nm}$ ) are observable on top of the second or third bilayer and can be attributed to discontinuations in the lipid membrane. The physiological electron transfer of this biomimetic system was probed with cyclic voltammetry in our recent publication. ${ }^{1}$ The appearance of a reversible redox reaction between cyt-c (from horse heart) and the surface-tethered $\mathrm{CcO}$ proves the oriented attachment and the functional integrity of the reconstituted $\mathrm{CcO}$. Within all our TM-AFM experiments, we found neither indication of tip induced surface modifications nor time induced degradation for at least $24 \mathrm{~h}$. Attempts to image these surfaces by contact mode AFM revealed serious surface distortions induced by the tip.

In summary, we monitored the resulting surface of the oriented incorporation of $\mathrm{CcO}$ into electrode supported lipid bilayer at four crucial stages with molecular resolved scanning probe microscopy. We were able to reveal (1) the formation of a densely packed monolayer of DTSB, (2) the 946.

(16) Tihova, M.; Tattrie, B.; Nicholls, P. Biochem. J. 1993, 292, 933-

(17) Wang, K.; Geren, L.; Zhen, Y.; Ma, L.; Ferguson-Miller, S.; Durham, B.; Millett, F. Biochemistry 2002, 41, 2298-2304. 
a

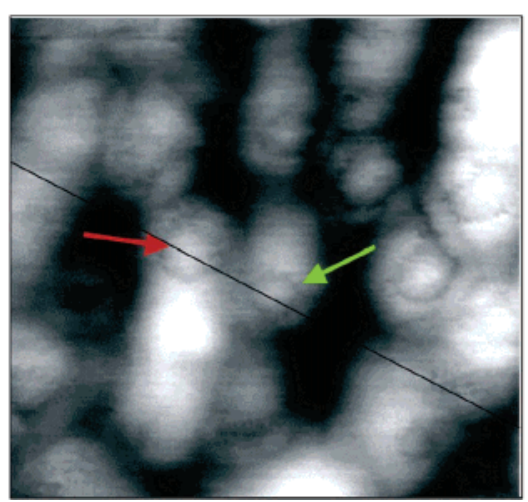

b

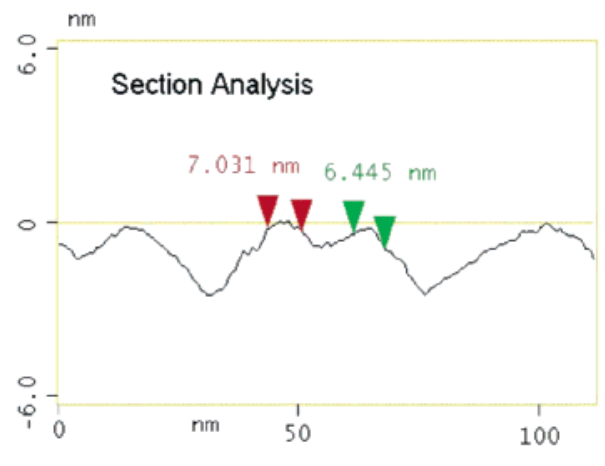

Figure 3. AFM image of adsorbed cytochrome $c$ oxidase on the Ni-NTA modified Au surface via the His-tag at the C-terminus of subunit I, (a) scan size $85 \mathrm{~nm}$ (left image) and (b) cross section as indicated in (a) (right image) presenting individual detergent solubilized proteins.

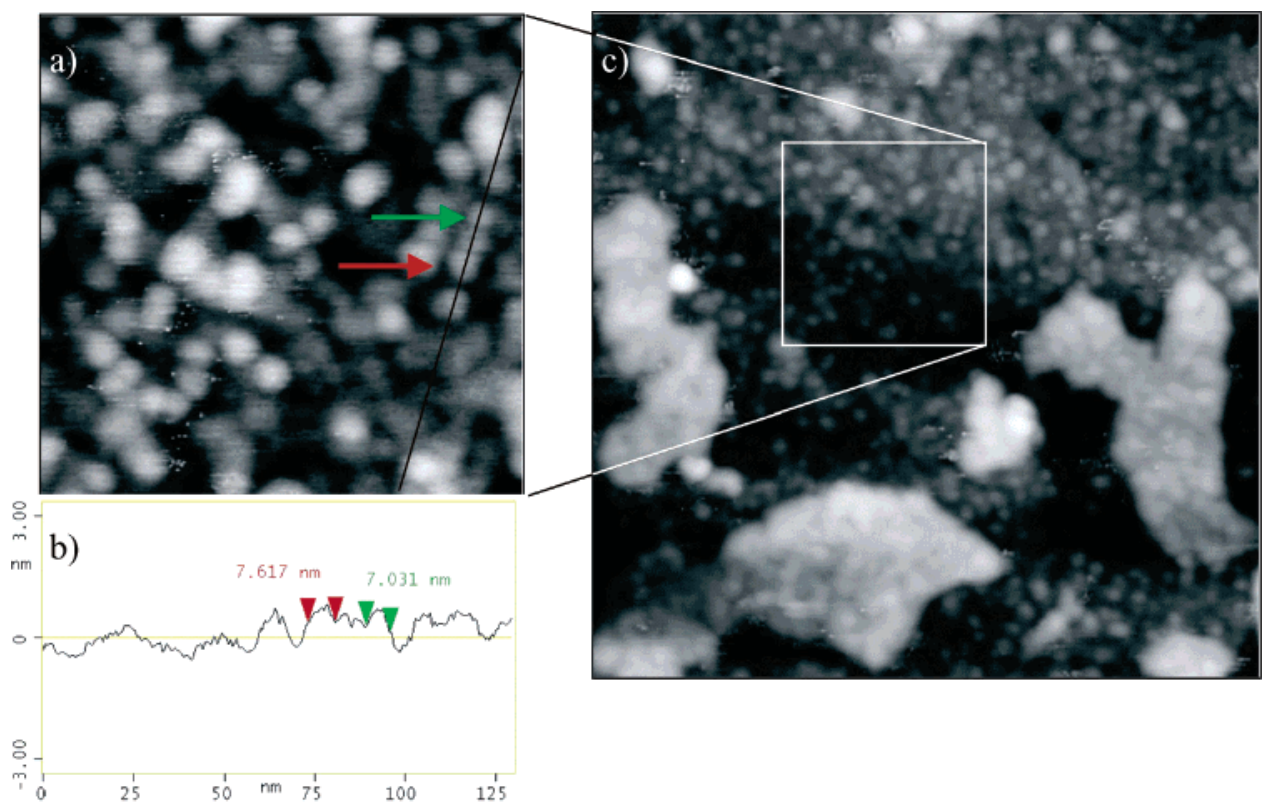

Figure 4. AFM image of surface-tethered cytochrome $c$ oxidase reconstituted into a lipid bilayer, (a) scan size $150 \mathrm{~nm}$ (left image), the red and green arrow indicates an aggregation of individual proteins, (b) cross section as indicated in (a), and (c) overview scan of (a), scan size $500 \mathrm{~nm}$ (right image).

covalent linkage of the NTA to the thiol anchored DTSB with almost $100 \%$ yield, (3) that the detergent solubilized oxidase is immobilized as monomers and small aggregates via the histidine tag, and (4) the reconstitution of the enzymes within the supported membrane. The amount of oxidase immobilized within the solid supported membrane was estimated to $33 \% \pm 4 \%$. The knowledge of the surface concentration permits kinetic measurements of the reaction between cytochrome $c$ and the $\mathrm{CcO}$ under various conditions. The accordance of measured physical dimensions of the enzyme with reported values indicates a protective character of detergent molecules and lipid membrane. Finally, the fabrication of well-oriented samples of membrane proteins may be employed for further studies of high-resolution microscopic imaging.

Acknowledgment. We are grateful to Prof. G. Büldt for continuous generous support. Financial support was by a grant from the Volkswagen Foundation ("Intra- and Intermolekulare Elektronenübertragung") to J.H.

LA051195X 